Eur. Phys. J. B 66, 259-269 (2008)

DOI: $10.1140 / \mathrm{epjb} / \mathrm{e} 2008-00419-\mathrm{y}$

\title{
Level-dynamic approach to the excited spectra of the Jahn-Teller model - kink-train lattice and 'glassy' quantum phase
}

\author{
Eva Majerníková ${ }^{1,2, a}$ and S. Shpyrko ${ }^{2,3, b}$ \\ 1 Institute of Physics, Slovak Academy of Sciences, Dúbravská cesta, 84511 Bratislava, Slovak Republic \\ 2 Department of Optics, Palacký University, Tř. 17. listopadu 50, 77207 Olomouc, Czech Republic \\ 3 Institute for Nuclear Research, Ukrainian Academy of Sciences, pr. Nauki 47, Kiev, Ukraine
}

Received: 12 June 2008 / Received in final form: 30 September 2008

Published online 20 November 2008

\begin{abstract}
The dynamics of excited phonon spectra of the $E \otimes e$ Jahn-Teller (hereafter, JT) model mapped onto the generalized Calogero-Moser $(\mathrm{gCM})$ gas of pseudoparticles implies a complex interplay between nonlinearity and fluctuations of quasiparticle trajectories. A broad crossover appears in a pseudotime (interaction strength) between the initial oscillator region and the nonlinear region of the kink-train lattice as a superlattice of the kink-antikink gCM trajectories. The local nonlinear fluctuations, nuclei (droplets) of the growing kink phase arise at the crossover, forming a new intermediate droplet "glassy" phase as a precursor of the kink phase. The "glassy" phase is related to a broad maximum in the entropy of the probability distributions of pseudoparticle accelerations, or level curvatures. The kink-train lattice phase with multiple kink-antikink collisions is stabilised by long-range correlations when approaching a semiclassical limit. A series of bifurcations of nearest-level spacings were recognised as signatures of pre-chaotic behaviour at the quantum level in the kink phase. Statistical characteristics can be seen to confirm the coexistence within all of the spectra of both regularity and chaoticity to a varying extent (nonuniversality). Regions are observed within which one of the phases is dominant.
\end{abstract}

PACS. 31.30.-i Corrections to electronic structure - 63.22.+m Phonons or vibrational states in lowdimensional structures and nanoscale materials - 05.45.-a Nonlinear dynamics and chaos $-34.10 .+\mathrm{x}$ General theories and models of atomic and molecular collisions and interactions

\section{Introduction}

The analysis of irregularities of quantum spectra is essentially the search for fingerprints of chaotic behaviour at the quantum level. It is especially delicate for models lacking a reasonable semiclassical limit due to the presence of quantum tunneling or nonadiabatic fluctuations [1. It is equally delicate for systems with a combination of regular and chaotic phase space 2. Previous studies have shown that nonadiabatic fluctuations act to stabilise the ground state, causing the ground state potential to become highly nonlinear [3. In this paper we shall investigate the consequences of the interplay of nonadiabatic fluctuations due to level correlations and nonlinearity in complex excited spectra of the $\mathrm{E} \otimes \mathrm{e}$ JT electron-phonon model in the situation of increasing interaction strength.

The JT model, with one control parameter of electronphonon interaction strength, can be mapped onto a fully integrable classical many-body system. This system is observed to experience repulsive long-range interactions known

\footnotetext{
a e-mail: eva.majernikova@savba.sk

b e-mail: serge_shp(at)yahoo.com
}

as the generalized Calogero-Moser gas ( $\mathrm{gCM})$ of Coulomb interacting pseudoparticles $[4,5,6,7$. This method provides a bridge to a statistical description of the system and thus appears to be a basis for differentiating between regularity and chaos in some systems [8,9,10, It is worth noting that the statistical description applies only for models with appropriate Gibbs measures.

In this paper, the authors seek to numerically and analytically examine level dynamics of levels as functions of the parameter (electron-phonon coupling as a pseudotime) in pseudospace. Probabilistic considerations enable determination of a measure of stochasticity within this irregular and highly non-universal model, in the context of earlier analyses of the nearest level spacing probability distributions [11. Besides the numerical analysis we shall apply an alternative approach of level dynamics. We shall present a related approximate analytical calculation to shed light on spectral structures, the physical mechanisms of their formation and the crossover between them. One advantage of this approach is the ability to classically represent quantum fluctuations and examine nonlinearity of the originally quantum system. 
E. Majerníková, S. Shpyrko: Level dynamics approach to the Jahn-Teller model

Excited energy levels $E_{n}(\alpha), n=1, \ldots, N$ of a quantum Hamiltonian in the form $H(\alpha)=H_{0}+\alpha V$ can be considered as dynamic coordinates of $N$ interacting classical pseudo-particles moving in a pseudo-time $\tau \equiv \alpha$, $E_{n}(\alpha) \equiv x_{n}(\tau)$. The gCM set of equations equivalent to the quantum mechanical problem $H(\alpha)|\phi\rangle=E_{n}(\alpha)|\phi\rangle$ reads 4,5 .

$$
\begin{gathered}
\mathrm{d} x_{n} / \mathrm{d} \tau=p_{n}(\tau), \quad \frac{\mathrm{d} p_{n}}{\mathrm{~d} \tau}=2 \sum_{m(\neq n)} \frac{L_{n m} L_{m n}}{\left(x_{m}-x_{n}\right)^{3}}, \\
\frac{\mathrm{d} L_{m n}}{\mathrm{~d} \tau}=\sum_{l \neq(m, n)} L_{m l} L_{l n}\left[\frac{1}{\left(x_{n}-x_{l}\right)^{2}}-\frac{1}{\left(x_{m}-x_{l}\right)^{2}}\right],
\end{gathered}
$$

where

$$
\begin{aligned}
& L_{m n}(\tau)=\left(x_{n}(\tau)-x_{m}(\tau)\right) \cdot V_{m n}(\tau)=-L_{n m}, \\
& p_{n} \equiv\langle n(\tau)|V| n(\tau)\rangle=V_{n n}(\tau), V_{n m}(\tau)=\langle n(\tau)|V| m(\tau)\rangle .
\end{aligned}
$$

This system is known to be completely integrable [6, 7]. Hence, it possesses as many integrals of motion as it has independent variables. The most important two additive integrals of motion are the total "energy" (the classical Hamiltonian which generates the above equations of motion (1))

$$
E=\frac{1}{2} \sum_{n=1}^{N} p_{n}^{2}-\sum_{1 \leq j<k \leq N} V_{j k}^{2}(\tau) \log \left|x_{j}-x_{k}\right|
$$

and the total "angular momentum" 5]

$$
Q=\sum_{1 \leq j<k<\leq N} L_{j k}^{2}
$$

The first or both integrals of motion were used to develop the statistical description of the gCM system by an appropriate choice of canonical ensemble and corresponding Gibbs measures [5,12.

Equation (3) allows us to understand the system as a two-dimensional Coulomb plasma of non-local time dependent pseudocharges $V_{j k}(\tau)$. The respective set of equations alternative to the equations for $L_{m n}$ is implied by (1) and (2) to yield [8]

$$
\begin{aligned}
\frac{\mathrm{d} V_{m n}}{\mathrm{~d} \tau}= & \sum_{l \neq(m, n)} V_{m l}(\tau) V_{l n}(\tau)\left[\frac{1}{x_{m}-x_{l}}+\frac{1}{x_{n}-x_{l}}\right] \\
& -V_{m n}(\tau) \frac{p_{m}-p_{n}}{x_{m}-x_{n}} .
\end{aligned}
$$

The formalism of level dynamics (1.5) may be applied to the excited phonon spectrum of the $\mathrm{E} \otimes \mathrm{e}$ JT Hamiltonian 3, 11

$$
H=\left(b_{1}^{\dagger} b_{1}+b_{2}^{\dagger} b_{2}+1\right) I+\alpha\left(b_{1}^{\dagger}+b_{1}\right) \sigma_{z}-\alpha\left(b_{2}^{\dagger}+b_{2}\right) \sigma_{x} .
$$

In this equation, $b_{1,2}$ represent boson (phonon) operators of two oscillators with the frequency $\Omega=1, \sigma_{x}=$ $\left(\begin{array}{ll}0 & 1 \\ 1 & 0\end{array}\right), \sigma_{y}=i\left(\begin{array}{cc}0 & -1 \\ 1 & 0\end{array}\right), \sigma_{z}=\left(\begin{array}{cc}1 & 0 \\ 0 & -1\end{array}\right)$ are Pauli matrices, $I$ is the unit matrix, $\alpha$ is the coupling constant between the electron and phonon modes. The $2 \times 2$ matrix form accounts for two electron levels. According to the above, the model (6) with one non-integrability parameter $\alpha\left(H(\alpha)=H_{0}+\alpha V\right)$ and the integrable part $H_{0}$ is suitable for gCM mapping (1) to be applied. It is worth noting that the case containing different frequencies of the phonon modes 1 and 2 can easily be transformed into a case with two different coupling constants $\alpha \neq \beta$ in the two last terms of (6). This was investigated in a series of previous papers [3, 13, 14. However, the level dynamics approach for systems with several nonintegrability parameters is much more complicated [7,15]. Analysis reveals non-trivial gauge structures induced by multidimensional parameter space.

The JT model (6) consists of two degenerate electron levels coupled with two vibron modes of different symmetry against reflection. It is a typical representation of a nonintegrable nonadiabatic system posessing no reasonable semiclassical limit. The model has been intensively studied over time [3, 10, 16, 17] and is known to be a rich source of understanding for physical properties and consequences, for both heuristic interest and practical applications. The rotationally symmetric E $\otimes \mathrm{e}$ model, besides of the common $S U(2)$ reflection symmetry, has one additional constant of motion - the conserved angular momentum $\hat{J}=i\left(b_{1} b_{2}^{+}-b_{1}^{+} b_{2}\right)-\frac{1}{2} \sigma_{y}$ with eigenvalues $j=1 / 2,3 / 2, \ldots$ Hence, in the following, the representation of the definite quantum number $j$ will be used which appears to be an additional parameter of the model [11. To illustrate the typical properties of the gCM gas we have numerically solved the set of gCM dynamical equations (1), (2) and (5) for the system (6). The initial conditions $V_{m n}(0)$ for the model $(6)$ are specified from the set of equations for the excited levels of the model. It consists of pairs of even and odd levels $E_{n}, n \equiv 2 n_{r}, 2 n_{r}+1$, $n_{r}=0,1, \ldots$ (main quantum number) determined by the well known tridiagonal matrix equations for energy $E$

$$
\begin{aligned}
& \left(E_{2 n_{r}}^{0}-E\right) c_{2 n_{r}}+\alpha\left(f_{n_{r}, n_{r}} c_{2 n_{r}+1}+f_{n_{r}, n_{r-1}} c_{2 n_{r}-1}\right)=0,(7) \\
& \left(E_{2 n_{r}+1}^{0}-E\right) c_{2 n_{r}+1}+\alpha\left(f_{n_{r}, n_{r}} c_{2 n_{r}}+f_{n_{r+1}, n_{r}} c_{2 n_{r}+2}\right)=0 .
\end{aligned}
$$

In this equation, $E_{n}^{0}$ represent the energies of unperturbed harmonic oscillators; $\left\{c_{n}\right\}$ are components of the wave function in the harmonic oscillator representation $\left(H_{0}\right.$ is diagonal). The perturbations terms in (7) are represented by the matrix elements [11,16]

$$
\begin{aligned}
& V_{2 n+1,2 n}(0) \equiv f_{n_{r} n_{r}}=\sqrt{2} \sqrt{n_{r}+1+|j-1 / 2|}, \\
& V_{2 n-1,2 n}(0) \equiv f_{n_{r} n_{r}-1}=-\sqrt{2} \sqrt{n_{r}}
\end{aligned}
$$

giving the values of pseudocharges (5) at $\tau=0$. 


\section{GCM dynamics of the Jahn-Teller excited spectra. Numerical results}

In this section we present a numerical solution for the set of gCM equations (1 2) for several values of rotational quantum number $j$. The initial conditions $x_{n}(0)=E_{n}^{(0)}$, $p_{n}(0)=0, V_{2 n+1,2 n}(0), V_{2 n-1,2 n}(0)$ are given by (8). Here and in subsequent sections the index $n$ labels the energy levels in increasing order while $n_{r}$ and $j$ represent the quantum numbers of the initial unperturbed oscillators [11. Typically the reduced sets of equations for twenty levels were examined. In order to confine the levels we imposed boundary conditions rendering the first and last levels unmovable, in other words, replacing the first and last pseudo-particle by the pseudo-particles of infinite mass. The examples of numerical solutions to $\mathrm{gCM}$ equations are shown in Figures 1, 2 for three sets of variables $\left(x_{n}, p_{n}\right.$, $\left.V_{m n}\right)$ involved. The solutions for energy levels not close to artificial interval boundaries locally effectively reproduce the numerical solutions for energy levels as functions of $\alpha$ obtained via the diagonalisation of the Hamiltonian matrix (7) (see [11, Fig.1). There the exact level spectrum is illustrated for two different domains introduced as "oscillating" and "kink-lattice" domains of the spectrum. These roughly correspond to the different types of behaviour shown in Figures 1, 2, As the number of levels increases, so does the effectiveness of the solutions at reproducing numerical solutions via diagonalisation of the Hamiltonian.

In Figures 1, 2 one can recognise a complex dynamical behaviour of three coupled groups of excitations: at small $\alpha$ there are two groups of pseudo-particles (levels), even and odd, of weakly perturbed oscillations with opposite amplitudes (Fig. 1a) and related rapidly oscillating pseudo-momenta $p_{n}$ (Fig. 1b). After a small initial period the level degenerations are removed due to the creation of new inter-level correlations of more distant levels represented by the "pseudocharges" $V_{m n}(\tau)$ for $m>n+1$ (Eqs. (1, 2)). They emerge as the third (central) band in Figures 1, 2,c. With increasing $\alpha$ and $j$ the interference between the central and side bands increases, destroying the oscillatory behaviour of the levels $x_{n}$ and related momenta $p_{n}$. This scenario tends to the formation of a single stochastic central band of pseudocharges with increasing pseudotime (Fig.2). Moreover, within the single stochastic band appear very narrow windows of collision-free ballistic motion, with linear dependence on $x_{n} \sim v \alpha$ and $p_{n} \sim$ const. This may be attributable to the kink domain in the long time limit when the joint effect of the stochastic set of correlations (including the long-distance inter-level correlations (Fig. 3c)) vanishes, allowing a situation similar to free particle dynamics. We shall comment further on the structure of the spectra on the basis of the analytical results contained in Section 3

Correlations between the levels $\left(x_{n+i}, x_{n}\right), i=1,3,5$ (maps) for different model parameters and parts of the spectra are illustrated in Figure 3. In these figures the notation $x_{n}$ stands for the "reduced" part of the level energy with extracted energy of the level $E_{0}$ and "secular"
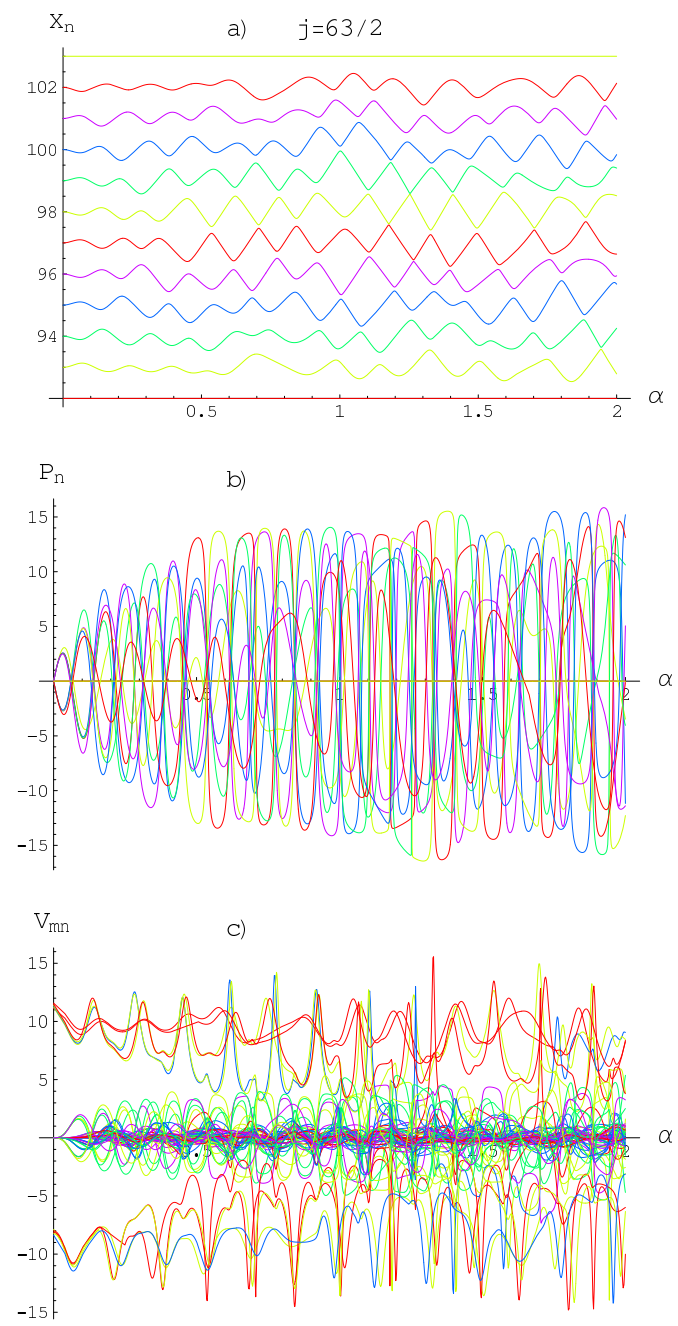

Fig. 1. Numerical solution of level dynamics equations (1)(2) in the $n_{r}>j$ part of the Jahn-Teller excited spectra for $j=63 / 2$ with artificial boundary conditions (see the beginning of Sect. 2). The levels from $n=93$ to $n=102$ are shown provided that those at $n=92$ and $n=103$ are kept fixed. (a), (b) show respectively "coordinates" $x_{n}$, and related momenta $p_{n} \equiv V_{n n}$, and (c) shows all the off-diagonal matrix elements $V_{m n}$ of the perturbation $\hat{V}$ for $m, n$ within the described range. The transition between the oscillatory (small $\alpha$ ) and the kink region with avoidings $\left(p_{n}=0\right)$ is marked by mixing of the $V_{m n}$ branches. The kink lattice region is characterised by strong mixing of three $V_{m n}$ branches. Narrow windows of weaker mixing at large $\alpha$ are apparent.

part $n-\alpha^{2}$ (Cf. Fig. 4 of 11). The semi-elliptic shape of the maps, related to the highest parts of the spectra, suggests that the correlations $x_{2 n+1} \cdot x_{2 n}$ have a strong effect with excluded space inside the ellipse (Figs. $3 a, 3$ b). This is a direct indication of level avoidings. The effect noticeably changes for different parts of the spectra and becomes more pronounced and unpredictable with increasing $\alpha$ and $j$. On the other hand, for extremely high $\alpha$ and $j$ close to the classical limit, the effect of correlations randomises the map so strongly that the excluded space substantially 


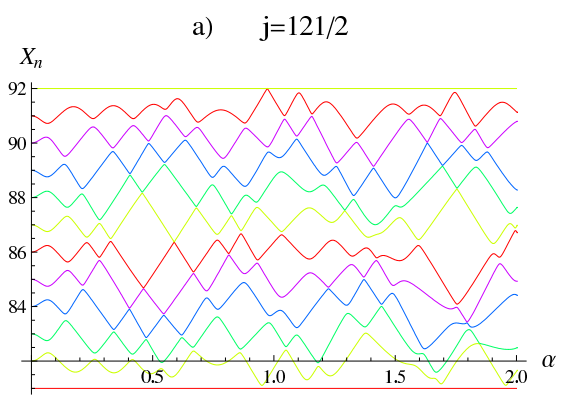

b)

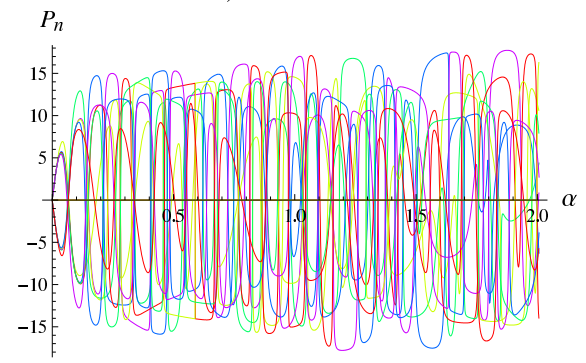

c)

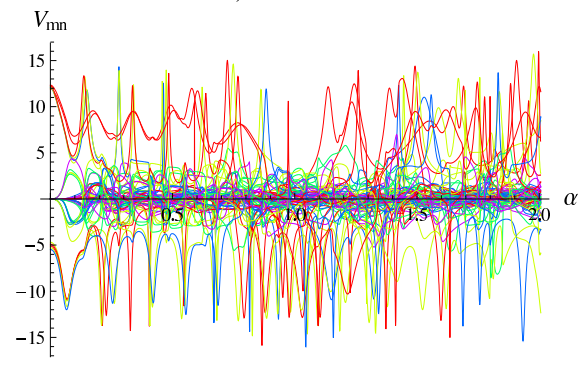

Fig. 2. The same for $j=121 / 2, n_{r}<j$. Note the kinklattice region $x_{n} \sim v \alpha$ at strong mixing of three $V_{n m}$ branches. Narrow segments of collisionless ballistic dynamics appear with linear time-dependent trajectories $x_{n}$ and constant momenta $p_{n}$ at $V_{m n}=0$. With increasing $\alpha$ the "oscillating" branches are absorbed by the central stochastic band.

shrinks to several regions of a possible fractal structure induced by the long-range correlations (Fig. $3 \mathrm{c}$ ).

\subsection{Probabilistic distributions of pseudo-particle characteristics}

A complementary approach to the developed dynamic (deterministic) picture can be provided by describing the system in terms of statistical distributions. The common ad hoc assumption is to introduce a canonical (or grand canonical) ensemble and an appropriate Gibbs measure in the phase space of all dynamical variables [5, 12] $\mathrm{d} M=$ $\exp (-\beta H) \mathrm{d} x_{n} \mathrm{~d} p_{n} \mathrm{~d} L_{m n}$. In the domain of developed quantum chaos, this approach was shown to reproduce the main results of random matrix theory (RMT). In particular, it is effective in predicting the form of the distribution of level spacings and level velocities. Similarly, it can be used for the investigation of the response of the energy spectrum to the change of nonintegrability parameter (that is for the distribution of pseudoparticle veloci- ties $v \equiv p_{n}=\mathrm{d} x_{n}(\tau) / \mathrm{d} \tau=V_{n n}(\tau)$ and their accelerations (level curvatures) $\left.P(K), K \equiv \mathrm{d}^{2} x_{n}(\tau) / \mathrm{d} \tau^{2}\right)$. In particular, in the domain of applicability of the RMT the distribution of the velocities should be Gaussian with the dispersion equal to the "temperature" parameter $1 / \beta$ in the Gibbs measure [8. The principal prediction for the distribution of the level curvatures is their asymptotic behaviour at large $K$ which has been shown universally to be of the form $P(K) \sim K^{-\nu}$ with $\nu=3,4,6$ for different kinds of ensembles 12 . The strongest assumption made in this approach is determining the form of the Gibbs measure, which implies that the system is in thermodynamic equilibrium. In the domains of undeveloped chaotic behaviour this assumption appears invalid. In particular, the statistical distributions $P(v)$ of the level velocities $v \equiv p_{n} \equiv V_{n n}(\tau)$ for fixed $j$ and $\tau \equiv \alpha$ for JT system are shown in Figure 4. They develop from an initial (at small $\alpha$ ) oscillation for large $n$ (upper part of the spectra, Fig. 1c, 4b) to strongly broadened oscillatory bands due to the stochastic velocities in the lower part of the spectra, Figure 2c, 4a. Two distinct bands of positive and negative velocities and the in-gap remnants of the oscillations seen in Figure $4 a$ are interpreted in Section 3

The non-Gaussian character of the distributions $P(v)$ in Figure 4 and $P(K)$, Figure 5 exposes the necessity of more correctly introducing the stochastic measure. As

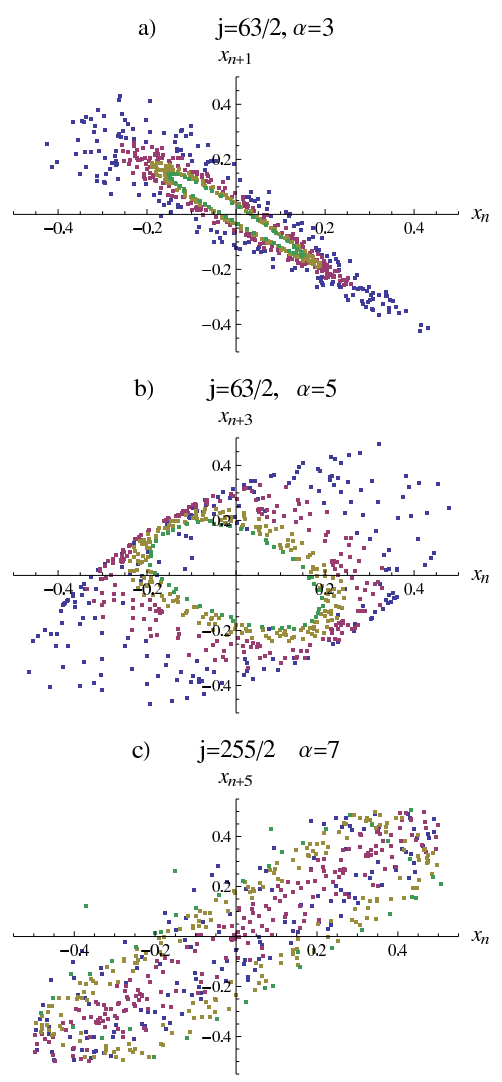

Fig. 3. Examples of maps of exact solutions $x_{n+i}$ vs $x_{n}$ for $i=$ $1,3,5$ for different $j$ and $\alpha$ and different parts of the spectra: levels with numbers 100-300 - blue, 300-500 - red, 500-700 yellow, 700-750 - green. 
the authors have previously suggested 11, the development of the distribution of $P(K)$ in "time" $\alpha$ has some features common with the diffusion process governed by the diffusion equation with additional telegraph term, i.e. $T \partial^{2} P / \partial \tau^{2}+\partial P / \partial \tau=D \partial^{2} P / \partial K^{2}$, where $D$ is the diffusion coefficient. The coefficient of the telegraph term accounts for memory effects. In particular, it is responsible for the appearance of the characteristic "wings" for large $K$ (Fig. 5b) which are not observed through the RMT approach. A correct explanation for these features requires introducing an equation for which the measure $\mathrm{d} M(\alpha)$ developes in time and only coincided with the Gibbs measure in the limit of RMT ("quantum chaos"). In view of the two-peak character of the probability distributions $P(K)$ we use their "entropies" $S_{K}=-\int P(K) \log P(K) \mathrm{d} K$ to characterize their properties rather than their dispersion characteristics [18].

The entropies $S_{K}$ of the probability distributions of the accelerations (curvatures) $P(K)$ averaged over successive intervals of 100 levels are plotted in Figure 6 as functions of pseudotime $\alpha$. The crossover between the two above described phases is evident. These figures show the broad maximum irregularity (stochasticity) in the region of the maximal mixing of three branches of the pseudocharges identified as the region of growing nonlinear fluctuations (formation of droplets) as a precursor phase of the kinktrain lattice (see subsequent section).
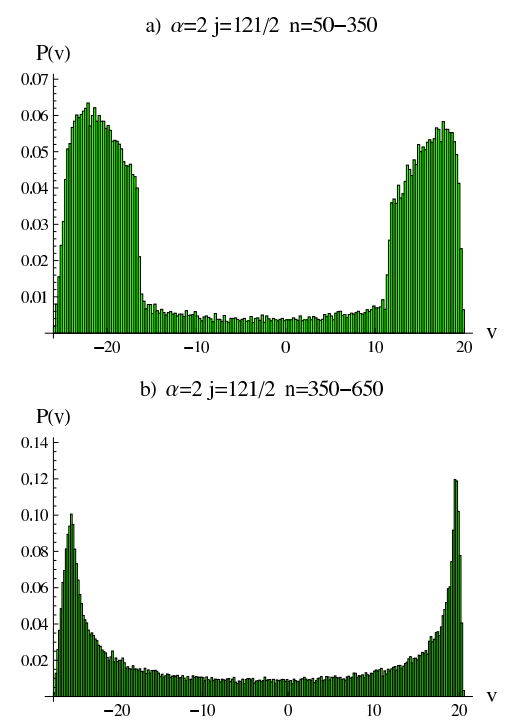

Fig. 4. Probability distributions of velocities of pseudoparticles $P(v), v \equiv V_{m m}(\tau)$. Peaked oscillator-dominated region in the upper part of the spectra (b); Stochasticity-dominated broad bands in the lower part of the spectra (a).

\section{GCM dynamics of the Jahn-Teller excited spectra. Approximate analytical approach}

The simplest analytical approach based on the approximation of constant (initial) values of matrix elements $V_{2 n+1,2 n}(\tau)$ and $V_{2 n-1,2 n}(\tau)$ can be applied for a small $\alpha$. It must be borne in mind that this approach neglects the dynamics of $V_{m n}(\tau)$, the correlations of more distant levels developed in the course of pseudotime (Figs,3,b,c). As a consequence our two-level approach includes only the dominant nearest neighbour interactions. To a limited extent this approximation allows for understanding the main features of the model and is satisfactorily consistent with our numerical results. This formulation recovers the local crossover between the anharmonic oscillator region and the complex nonlinear one.

Within the approximation of constant matrix elements in (2) it may be taken that $V_{2 n+1,2 n}(\tau) \approx V_{2 n+1,2 n}(0)=$ $f_{n n}\left(n_{r}, j\right), V_{2 n-1,2 n}(\tau) \approx V_{2 n-1,2 n}(0)=f_{n, n-1}\left(n_{r}, j\right)$ from (8) (in what follows we denote $j \equiv|j-1 / 2|$ for brevity). Then,

$$
\begin{aligned}
& L_{2 n+1,2 n}(\tau)=V_{2 n+1,2 n}(0)\left(x_{2 n+1}(\tau)-x_{2 n}(\tau)\right), \\
& L_{2 n-1,2 n}(\tau)=V_{2 n-1,2 n}(0)\left(x_{2 n}(\tau)-x_{2 n-1}(\tau)\right) .
\end{aligned}
$$

Since the number of phonons is not conserved the number of pseudoparticles (excited levels) in the system with Hamiltonian (6) is not constant. Therefore a two-level approximation of the gCM equations can be used, but only for small $\alpha$. Denote the corresponding levels as $x_{1}, x_{2}$. The origin may be set so that two levels $x_{1}, x_{2}$ are symmetric and separated by $x_{2}-x_{1} \equiv 2 x$; the effect of the other levels of the system can be accounted for by setting two bounding (fixed) levels, $x_{0}, x_{3}$ separated by a

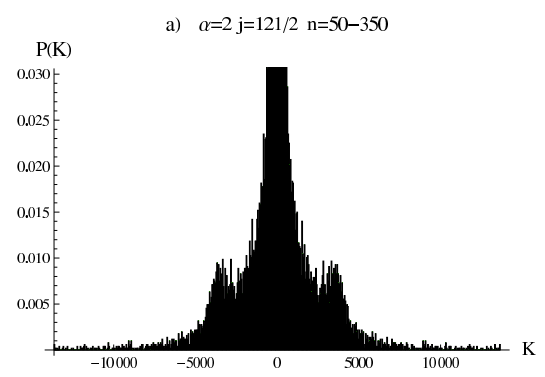

b) $\quad \alpha=2 \mathrm{j}=121 / 2 \mathrm{n}=650-950$

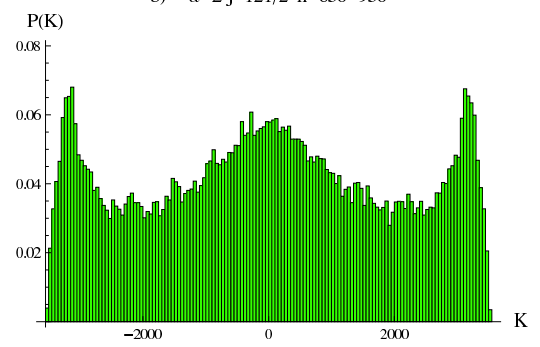

Fig. 5. Probability distributions $P(K)$ for $\alpha=2, j=121 / 2$, where $K=\partial^{2} x_{n} / \partial \tau^{2}$ is the level curvature (pseudoparticle acceleration). Note the two-peaked oscillator-dominated region in the upper part of the spectra (b). The stochasticity-dominated region in the lower part of the spectra exhibits (i) a prominent peak at $K=0$ which corresponds to the kinks ( $p=$ const, see Fig. 2b); (ii) characteristic for RMT prediction of asymptotic power-like dependence at large $K$. The side "wings" of the distributions are in contrast to the predictions of the RMT and indicate a presence of memory terms in the equation for the measure $\mathrm{d} M(\alpha)$. 

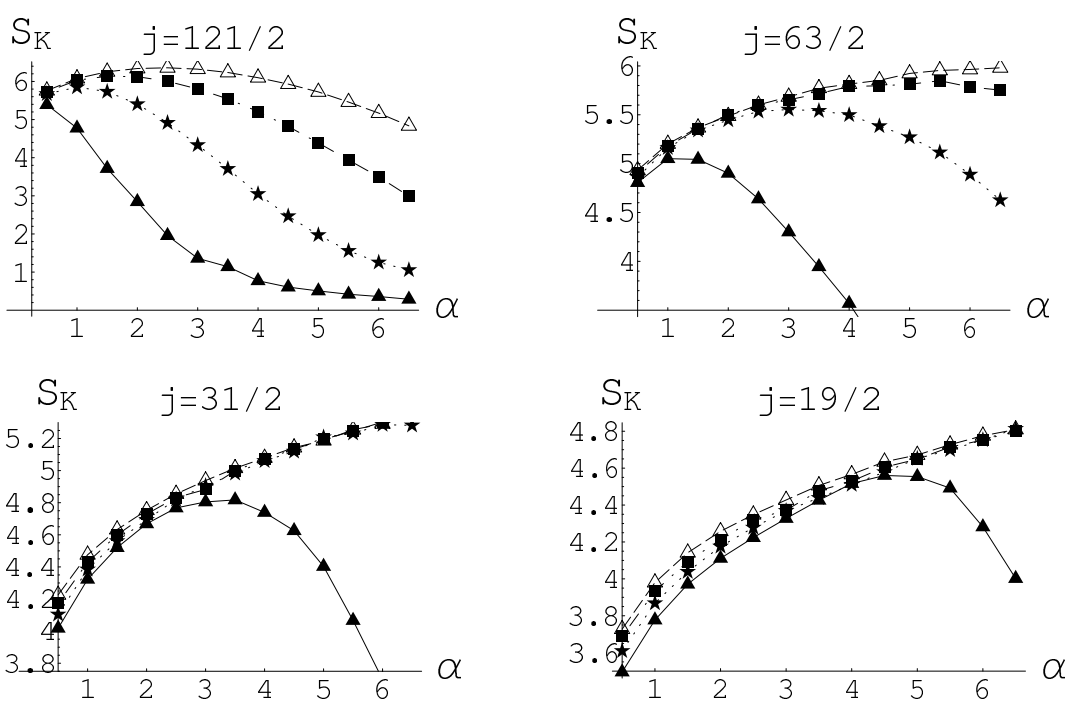

Fig. 6. Entropies $S_{K}$ of the probability distributions of the accelerations (curvatures) $P(K)$ averaged over successive intervals of 100 levels. The maximum irregularity (stochasticity) appears in the region with maximum mixing of three branches of the pseudocharges identified as the region of droplet formation and as a precursor phase of the kink-train lattice. Triangles - levels 50-150; stars: levels 150-250; squares: 250-350; open triangles: 350-450. The entropies are given with an arbitrary constant of the form $\Delta K \log \Delta K$ accounting for the finite sampling bins $\Delta K$ on the histograms such as shown in Figure 5 , which were the same $(\Delta K=10)$ for all graphs.

distance $x_{3}-x_{0}=3$. To simplify notations we denote $f_{n n} \equiv f_{0}, f_{n+1, n} \equiv f_{1}$. The set of gCM equations then yields

$$
\left\{\begin{array}{l}
\frac{1}{2} \frac{\partial p_{1}}{\partial \tau}=\frac{-f_{0}^{2}}{x_{2}-x_{1}}+\frac{f_{1}^{2}}{x_{1}-x_{0}} \\
\frac{1}{2} \frac{\partial p_{2}}{\partial \tau}=\frac{f_{0}^{2}}{x_{2}-x_{1}}-\frac{f_{1}^{2}}{x_{3}-x_{2}}
\end{array}\right.
$$

The equation for the separation $x$ of the two nearest trajectories reads

$$
\ddot{x}=-\frac{\partial V(x)}{\partial x}=\frac{2 f_{0}^{2}}{2 x}-\frac{2 f_{1}^{2}}{3 / 2-x},
$$

where $V(x)=-f_{0}^{2} \log x-2 f_{1}^{2} \log (1.5-x)$ is the onedimensional time-dependent Coulomb potential with $x_{e q}=$ $3 f_{0}^{2} / 2\left(f_{0}^{2}+2 f_{1}^{2}\right)$ as a point of the potential minimum where $V^{\prime}\left(x_{e q}\right)=0$.

The harmonic oscillations of the frequency $V^{\prime \prime}=2\left(2 f_{1}^{2}+\right.$ $\left.f_{0}^{2}\right)^{3} /\left(3 f_{0} f_{1}\right)^{2}$ become unstable at $V^{\prime \prime \prime}=0$, or when $f_{0}^{2}=$ $2 f_{1}^{2}$ or $n_{r} \approx j$. This condition determines the border between the harmonic oscillation region $\left(n_{r}>j\right)$ and the nonlinear region $\left(n_{r}<j\right)$ in this simplified model. The model generates the map illustrated in Figure 7. Since the subsequent clusters in it are independent, the whole map is completely determined by the phase shift of the oscillations from cluster to cluster (changing the parameter $n_{r}$ numbering the clusters). Resemblance to the corresponding exact results, Figure 3 a can be traced only for higher levels otherwise the simple two-level symmetric model completely ignores the level avoidings.

In order to proceed beyond the two-level harmonic approximation we shall account for the collective effect of neighbour levels with level-to-level variations of the "pseudocharges" $f_{m n}$. Let us define small fluctuations $\delta$ as $x_{2 n+1}-x_{2 n} \equiv 1+\delta_{2 n}$. The set of equations derived from (7) then reads

$$
\begin{gathered}
\frac{1}{2} \frac{\partial^{2} \delta_{2 n}}{\partial \tau^{2}}=\frac{2 f_{n n}^{2}}{1+\delta_{2 n}}-\frac{f_{n+1, n}^{2}}{1+\delta_{2 n+1}}-\frac{f_{n, n-1}^{2}}{1+\delta_{2 n-1}} \\
\frac{1}{2} \frac{\partial^{2} \delta_{2 n+1}}{\partial \tau^{2}}=\frac{2 f_{n+1, n}^{2}}{1+\delta_{2 n+1}}-\frac{f_{n+1, n+1}^{2}}{1+\delta_{2 n+2}}-\frac{f_{n n}^{2}}{1+\delta_{2 n}} .
\end{gathered}
$$

For sufficiently large $n, f_{n+1, n+1} \approx f_{n n}, f_{n+1, n} \approx f_{n, n-1}$, hence equations (12) imply the approximate relations $\delta_{2 n+1}$ $\approx \delta_{2 n-1}$ and $\delta_{2 n+2} \approx \delta_{2 n}$. Then the pairs of pseudoparticles (levels) represent an analogy of a dimerized system or a lattice with two atoms in a cell. It is worth noting that from (12) it follows that $\delta_{2 n}, \delta_{2 n+1}$ is a pair of reflectionsymmetric variables against the transformation $\tau \rightarrow i \tau$.

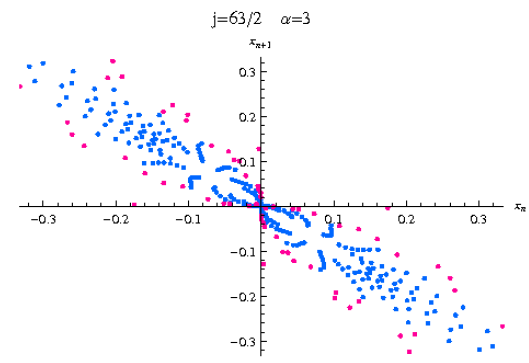

Fig. 7. Mapping of approximate solutions $x_{n+1}$ vs $x_{n}$ (as in Fig. 3 a) generated by the simple two-level model (10). The correspondence with the exact result in Figure 3 is satisfactory except in the near-zero part since the level avoidings are neglected in this model. 


\subsection{Structure of the nonlinear region and quantum phase transition-like nature of the crossover}

For increasing $\tau$ and $j$ the picture of "pseudocharges" $V_{m n}(\tau)$ (Eq. (3) and Fig.1) dramatically changes by the creation of new long-range components which form a central branch of highly irregular oscillations. A strong mixing between the short-range (two side-bands in Fig.1) and long-range $V_{m n}$ (central band) proceeds towards a limit of large j, when the side-bands disappear (Fig.2c). At large $\alpha$, the level dynamics is mostly affected by the central irregular branches of the "pseudocharges" $V_{m n}$. A simple approximate dynamic description of the crossover between oscillatory and nonlinear regions can be obtained from the dynamic equations (12) for the fluctuations, $\delta_{2 n}$ and $\delta_{2 n+1}$. We develop the terms on r.h.s. of equations (12) into a series up to the lowest order of nonlinearity $\delta^{3}$. In the following approximate calculations we shall move to a continuum approximation in the coordinate $n$ labeling the level system. If we extract the term

$$
\delta_{2 n+2}+\delta_{2 n}-2 \delta_{2 n+1} \rightarrow \frac{\partial^{2} \delta_{2 n+1}}{\partial n^{2}} \equiv \delta_{2 n+1}^{\prime \prime}
$$

and define $\delta_{2 n+1} \equiv 1+y, \delta_{2 n} \equiv x$, the equation for $\delta_{2 n+1}$ of (12) can be rewritten in a suitable form for further analysis,

$$
\begin{aligned}
& \frac{1}{2} \ddot{y}-f_{n+1, n+1}^{2} y^{\prime \prime}=f_{n+1, n+1}^{2}-f_{n n}^{2}+ \\
& 2\left(f_{n+1, n+1}^{2}-2 f_{n+1, n}^{2}\right) y-2 f_{n+1, n}^{2} y^{2}(y+2) \\
& -\left(f_{n+1, n+1}^{2}-f_{n n}^{2}\right) x-\left(f_{n+1, n+1}^{2}+f_{n n}^{2}\right)\left(x^{2}-x^{3}\right),
\end{aligned}
$$

where the index $n$ of $y_{n}$ has been omitted for simplicity. Using a periodicity condition $\bmod (y+2)=y$ the equation (14) is rewritten as follows:

$$
\frac{1}{2} \ddot{y}-f_{n+1 n+1}^{2} y^{\prime \prime}=-\frac{\partial V(y)}{\partial y},
$$

where the nonlinear potential

$$
\begin{aligned}
& V(y)=\frac{1}{2} f_{n+1 n}^{2} y^{4}-\left(f_{n+1, n+1}^{2}-2 f_{n+1, n}^{2}\right) y^{2}- \\
& \left(f_{n+1, n+1}^{2}-f_{n n}^{2}\right)(1-x) y+\left(f_{n+1, n+1}^{2}+f_{n n}^{2}\right) x^{2}(1-x) y
\end{aligned}
$$

indicates a crossover analogous to the quantum first order phase transition due to a small linear driving force $\sim y$, $(|x| \ll|y|$ by definition). With the simplification valid for large $n, f_{n n}^{2} \approx f_{n+1 n+1}^{2}, f_{n+1 n}^{2} \approx f_{n n-1}^{2}$ one can obtain a condition defining the dimerisation region $\delta_{2 n} \approx-\delta_{2 n-1}$ where equation (12) for $\delta_{2 n}$ yields an equation analogous to (15). In the case when the driving forces on the r.h.s. of (16) $(\sim y)$ are either mutually compensated or both tend to zero (large $j$ and $\left.n_{r}\right)$, that is, $\left(f_{n+1, n+1}^{2}-f_{n n}^{2}\right)(1-x) \sim$ $\left(f_{n+1, n+1}^{2}+f_{n n}^{2}\right) x^{2}(1-x) \ll f_{n n}^{2}$, equations (15) and (16) imply a crossover analogous to the quantum second order phase transition at $f_{n+1, n+1}^{2}-2 f_{n+1, n}^{2}=2\left(j-n_{r}\right)=0$. This coincides with the criterion for the crossover to nonlinear region from simple analysis of equation (11). For $j<n_{r}, f_{n+1, n+1}^{2}-2 f_{n+1, n}^{2}<0, V(y)=\frac{1}{2} f_{n+1, n}^{2} y^{4}-$ $\left(f_{n+1, n+1}^{2}-2 f_{n+1, n}^{2}\right) y^{2}$ becomes a single-well potential, and we identify the phase of anharmonic oscillators. At $j \geq n_{r}$ one has $f_{n+1, n+1}^{2}-2 f_{n+1, n}^{2}>0$. Here a double potential well of $V(y)$ opens, and correspondingly a new phase appears. Equation (15) with the driving force omitted acts as an analogy to a quantum second order phase transition for each level (trajectory) $n$. However, the small driving force in (16) changes this scenario before it vanishes at large $j$ and $\alpha \equiv \tau$. Examining equation (14) with accounting for a constant ( $y$-independent) driving force

$F=\left[-\left(f_{n+1, n+1}^{2}-f_{n n}^{2}\right)+\left(f_{n+1, n+1}^{2}+f_{n n}^{2}\right) x^{2}\right](1-x)>0$

results in

$\frac{1}{2} \ddot{y}-f_{n+1, n+1}^{2} y^{\prime \prime}-2\left(f_{n+1, n+1}^{2}-2 f_{n+1, n}^{2}\right) y+2 f_{n+1, n}^{2} y^{3}=-F$,

here, $F$ in equation (17) consists of two competing parts: the drift term due to the broken periodicity of the level spectra at finite $\tau$ and the "pressure" force from the neighbouring levels. If $F>0$ and $f_{n+1, n+1}^{2}-2 f_{n+1, n}^{2}>0$ it drives a first order quantum phase transition-like crossover generating nonlinear fluctuations. The equation of type (18) in the dimension $d>1$ has been investigated [19] as a prototype for a first order phase transition involving the coexistence of two phases, one phase represented by growing droplets (bubbles) spanned by the force $F$ in the sea of the other competing phase. In our case the dimension $d=1$; Nevertheless, there exists an exact solution to the normalized nonlinear equation of the type (18). It was found in the closed form of a nonlinear oscillation [20] which in our case reads

$$
y\left(w\left(2 \tau-\frac{n_{r}}{\sqrt{j}}\right)\right)=a \frac{n_{1}+\cos \left(w\left(2 \tau-\frac{n_{r}}{\sqrt{j}}\right)\right)}{n_{2}+\cos \left(w\left(2 \tau-\frac{n_{r}}{\sqrt{j}}\right)\right)},
$$

where

$$
\begin{gathered}
n_{1}=\frac{2-n_{2}^{2}}{n_{2}}, a^{2}=\frac{f_{n+1, n+1}^{2}-2 f_{n+1, n}^{2}}{f_{n+1, n}^{2}} \frac{n_{2}^{2}}{2+n_{2}^{2}}>0, \\
w^{2}=\left(f_{n+1, n+1}^{2}-2 f_{n+1, n}^{2}\right) \frac{n_{2}^{2}-1}{n_{2}^{2}+2}, \\
a=\frac{F}{f_{n+1, n+1}^{2}-2 f_{n+1, n}^{2}} \geq 0 .
\end{gathered}
$$

The "droplet" fluctuation (19) is a traveling periodic non-sinusoidal oscillation moving with velocity $v \approx 2 \sqrt{j}$. The amplitude $a$ of the fluctuation $y$ is spanned by the driving field $F$. This force grows when $x\left(x \leq x_{\max }=\right.$ $\left.1 / \sqrt{2\left(j+n_{r}\right)}\right)$ decreases until the energy of the excitation (19) reaches the energy of the kink, where $v \approx 2 \sqrt{j}$ is the minimum velocity of the kink. If $j$ increases, $j \gg n_{r}$, then $x \rightarrow 0$ and $a \rightarrow 0\left(a \rightarrow 1 /\left(j-n_{r}\right)\right)$, so that the 
intermediate "droplet" glassy phase transforms into the more stable kink lattice phase (see below).

Simultaneously, with increasing $j$ and $\alpha$ the dimerization condition $\bmod (y+2)=y$ fails (Fig. 2), $f_{n+1, n+1}^{2} \approx$ $f_{n, n}^{2}$ and the driving force $F$ vanishes $\left(x_{\max } \rightarrow 0\right)$. The equation (14) up to second order terms tends to

$$
\frac{1}{2} \ddot{y}-f_{n+1, n+1}^{2} y^{\prime \prime}=2\left(f_{n+1, n+1}^{2}-2 f_{n+1, n}^{2}\right) y-4 f_{n+1, n}^{2} y^{2} .
$$

If we move to imaginary space $\zeta=i(v \tau-n)$, then the solution of (20) yields a traveling pulse

$$
\begin{aligned}
& y=\frac{3\left(f_{n+1, n+1}^{2}-2 f_{n+1, n}^{2}\right)}{4 f_{n+1, n}^{2}} \\
& \times \cosh ^{-2}\left(\left[\frac{f_{n+1, n+1}^{2}-2 f_{n+1, n}^{2}}{2 f_{n+1, n+1}^{2}-v^{2}}\right]^{1 / 2}\left(\zeta-\zeta_{0}\right)\right) .
\end{aligned}
$$

From the definition of $\delta_{2 n+1}$ we have $x_{2 n+2}-x_{2 n+1} \approx$ $\partial x_{2 n+1} / \partial n=1+\delta_{2 n+1}=2+y$ and equation (21) implies a kink-shaped profile,

$$
\begin{aligned}
& x_{2 n+1}=\text { const }+2 n+ \\
& \frac{3}{4 f_{n+1, n}^{2}}\left[\left(f_{n+1, n+1}^{2}-2 f_{n+1, n}^{2}\right)\left(2 f_{n+1, n+1}^{2}-v^{2}\right)\right]^{1 / 2} \\
& \times \tanh \left(\left[\frac{f_{n+1, n+1}^{2}-2 f_{n+1, n}^{2}}{2 f_{n+1, n+1}^{2}-v^{2}}\right]^{1 / 2}\left(\zeta-\zeta_{0}\right)\right),(22)
\end{aligned}
$$

where $v^{2}<2 f_{n+1, n+1}^{2}=v_{\max }^{2}$ is the velocity of the travelling kink profile and

$$
L=\frac{1}{2}\left[\frac{2 f_{n+1, n+1}^{2}-v^{2}}{f_{n+1, n+1}^{2}-2 f_{n+1, n}^{2}}\right]^{1 / 2}
$$

is the kink width. The time of collision can be estimated from $v \tau_{c}=2 L>\left(2 f_{n+1, n+1}^{2} /\left(f_{n+1, n+1}^{2}-2 f_{n+1, n}^{2}\right)\right)^{1 / 2} \sim$ $\sqrt{\left(2\left(j+n_{r}\right) /\left(j-n_{r}\right)\right)}, j>n_{r}$. Hence, the collision time close to the semiclassical limit yields $\tau_{c} \rightarrow 1 / \sqrt{2 j}$.

Equation (15) with omitted linear terms in the potential (16) represents a nonintegrable member of a family of Klein-Gordon equations, that is, $\Phi^{4}$ equation known to exhibit kink, antikink and a double kink solitary solutions 23. The solution (22) represents the tunneling between two nearest neighbor trajectories. Indeed, for large $j\left(f_{n, n}^{2}>2 f_{n+1, n}^{2}\right.$ or $\left.j>n_{r}\right)$ it gets a form of a propagating nonlinear solitary excitation, a kink (antikink), in the imaginary space $y\left(\zeta-\zeta_{0}\right), \zeta=i(v \tau-n)$, where $\zeta_{0}=i\left(v \tau_{0}-n_{0}\right)$ restores the translation symmetry.

The transition to the imaginary space effectively reverts the sign of the potential, that is, a pseudoparticle turns to the tunneling domain and changes its parity (cf. the mirror symmetry of equations (12)). For example, $\delta_{2 n+1}\left(v_{1}\right) \rightarrow \delta_{2 n}\left(v_{1}\right), \delta_{2 n}\left(v_{2}\right) \rightarrow \delta_{2 n+1}\left(v_{2}\right)$. As a result, the trajectories (pseudoparticles) interchange their velocities $v_{1}, v_{2}: \delta_{2 n}\left(v_{2}\right) \rightarrow \delta_{2 n}\left(v_{1}\right)$ and $\delta_{2 n+1}\left(v_{1}\right) \rightarrow \delta_{2 n+1}\left(v_{2}\right)$ when transferring from the real to the imaginary space and vice versa. This scenario can be understood as a series of kink-antikink collisions related to two subsequent levels.

Thus, the nonlinear phase represents the gCM trajectories as a kink-antikink chain structure apparent in Figure 2. Such a solution qualitatively agrees with the results of authors dealing with similar systems within the gCM level dynamics approach $[6,8,21,22$ predicting the "soliton"-like structures.

The real configuration of the level system under consideration is described by the discrete label $n$. In recent years more realistic discrete lattice versions (13) of KleinGordon equations have been in the focus of interest; they show a rich variety of behaviour. Besides the resonance energy exchange at kink-antikink collisions, a recently discovered aspect of this behaviour is the chaotic scattering upon certain conditions and fractal behaviour of velocities after collision strongly dependent on initial conditions 24, 25, 26, 27,28. The kink-antikink collisions in our system might follow this scenario of initiating the chaotic behaviour and fractal structure at large $j$, Figure $3 \mathrm{c}$.

In the case of $\partial^{2} \delta / \partial \tau^{2}=0$ we have $p_{n}=$ const so that the value of the curvature $K_{n}=\partial^{2} x_{n} / \partial \tau^{2}=0$ which corresponds to the peak in Figure $5 \mathrm{a}$ represents the kinks. One has $x_{2 n+1}=x_{2 n}+v \Delta \tau$ (here $\Delta \tau$ is the time distance between two kinks) and from (12) one finds

$$
\begin{gathered}
\delta_{2 n+1}+\frac{f_{n, n-1}^{2}}{f_{n+1, n}^{2}} \delta_{2 n-1} \approx \\
\frac{-2 f_{n n}^{2}+f_{n+1, n}^{2}+f_{n n-1}^{2}}{f_{n+1, n}^{2}}+\frac{2 f_{n n}^{2}}{f_{n+1 n}^{2}} \delta_{2 n}\left(1-\delta_{2 n}\right)
\end{gathered}
$$

and

$$
\begin{aligned}
& \delta_{2 n+1} \approx \frac{2 f_{n+1, n}^{2}-f_{n+1, n+1}^{2}-f_{n n}^{2}}{2 f_{n+1, n}^{2}}+ \\
& +\frac{f_{n+1, n+1}^{2}}{2 f_{n+1 n}^{2}} \delta_{2 n+2}\left(1-\delta_{2 n+2}\right)+\frac{f_{n n}^{2}}{2 f_{n+1 n}^{2}} \delta_{2 n}\left(1-\delta_{2 n}\right) .
\end{aligned}
$$

Equations (23) and (24) are reminiscent of the logistic equation $x_{n+1}=A x_{n}\left(1-x_{n}\right)$ which implies a known scenario for the transition to the chaotic region for $A=$ $A_{\text {crit }}=3.56994 \ldots$ In our case $A \sim\left(f_{n+1, n+1}^{2}+f_{n n}^{2}\right) / 2 f_{n+1, n}^{2}$ $\sim\left(n_{r}+|j-1 / 2|\right) / n_{r}$. The ranges of values of $j$ and $n_{r}$ considered above give the values of $A \geq 2$ close to 2 from above, that is, from the subcritical region. The hallmark of chaos at the quantum level can be identified as a prechaotic region of the series of bifurcations of the intermediate glassy phase.

The pre-chaotic behaviour applies to the medium region of values of $j$ and $n_{r}$ between the domains of weak coupling with $j<n_{r}$ (dimerized pairs of damped oscillators) and strong coupling with $j \gg n_{r}$. The description via initial quantum numbers at $\alpha=0, n_{r}$ and $j$ related to the radial and rotational degrees of freedom of the unperturbed oscillators, is kept by the mapping onto the classical gCM gas. For large $j, j \gg n_{r}$, the short-range 
correlations are strongly weakened while the long-range correlations dominate, as well as fluctuating around zero (Fig. 2c).

The kink-antikink chain level structure describes the special kind of self-avoiding motion of the gCM trajectories in the integrability parameter $\alpha$ as a pseudotime. Alternatively, one can also consider the levels as moving along almost straight lines broken by small intervals where they undergo "collisions" with neighbouring levels. They can be clearly visualized as a series of propagating pulses through the series of gCM pseudoparticles, along straight lines of positive and negative velocities. The apparent regularity of this structure allows us to introduce the idea of a superlattice of excitations in the space of gCM pseudoparticles. It is this new structure which we call the kink-train lattice, which may represent a new quantum chaotic phase in certain segments of the JT spectra, created by the kinkantikink collision mechanism described above.

A multiplicity of kinks and antikinks (solitary waves) travelling through gCM particles may be readily observed in Figure 1b of the another paper on the subject by these authors 11. It is recognisable in the numerical gCM simulations in Figure $2 a$, and in the statistical distribution of velocities of gCM pseudoparticles, Figure 4. The lack of symmetry of the positive and negative velocities against zero illustrates the driving of the lattice by a field superimposed by the broken periodicity, in turn implied by $f_{n+1, n+1}>f_{n n}$ over the whole lattice.

The trajectories forming the kink-antikink train lattice move as almost free particles between the collisions, with velocities of opposite sign distributed within two bands (Fig. 4a). The remnants of oscillations within the gap demonstrate the coexistence of both oscillating and kink phases. The long-range order developed at large $\alpha$ stabilises the almost periodic kink-train lattice (Fig. 6).

It is worth noting that for $j>n_{r}$ the effective values of the logistic map constant $A$ can reach the values above the critical $A_{c}$ for some $n_{r}$. This indicates chaotic behaviour of the level spacings. However, the cumulative spectra (including all $j$ ) impose intersections of the trajectories because of the multiple degeneracy in $j$ which will dominate and imply, for example, the Poisson-like probability behaviour of the nearest neighbour level spacing distribution $P(s)$ at $s \rightarrow 013$. Hence we can conclude that a coexistence of regular and irregular nonuniversal behaviour is characteristic for the excited spectra of the present model and in the whole extent of the interaction parameter.

\subsection{Semiclassical approximation}

One possible way of identifying the quantum chaoticity is to provide an analogy to the behaviour of the system at a classical or semiclassical level. The peculiarity of the class of electron-phonon systems is the marked ambiguity of passing to a semiclassical approximation from an initial set of Heisenberg equations implied by Hamiltonian (6). The common algorithm, decoupling products of quantum observables and replacing them by the classical ones, can be performed in different ways. The problem of moving to a semiclassical approximation was touched in a previous paper 11. Nevertheless, the investigation of trajectories of semiclassical observables reveals a picture of classically chaotic domains. These at least present a qualitative correlation to the picture of "quantum chaotic" behaviour outlined above. Respective equations of motion are implied by the semiclassical version of the Hamiltonian (6) in the form

$$
H=\frac{1}{2}\left(P_{1}^{2}+P_{2}^{2}+Q_{1}^{2}+Q_{2}^{2}\right)+\alpha Q_{1} z-\alpha Q_{2} x .
$$

Here, we introduced the coordinates of phonons $Q_{i}$, $P_{i}$ and of the electron $x \equiv\left\langle\sigma_{x}\right\rangle, y \equiv\left\langle\sigma_{y}\right\rangle, z=\left\langle\sigma_{z}\right\rangle$. The electron coordinates satisfy the condition $x^{2}+y^{2}+$ $z^{2}=1$ for the Bloch sphere. Additionally, besides the conservation of the energy (25), the angular momentum $J=Q_{1} P_{2}-Q_{2} P_{1}-y$ is a constant of motion. As a result, the system has four degrees of freedom.

For illustrative purposes, in Figure 8 we present the Fourier spectrum of the semiclassical trajectory $Q_{1}(t)$ (a picture for $Q_{2}$ looks similar) for different values of the energy $E$ of a system. The middle part of the energy interval (corresponding approximately to the intermediate part of the quantum spectrum with the strongest mixing of three effective potential wells) shows the marked domain of classical chaos. Meanwhile, the motion of the classical trajectories at low and high energies is noticeably regular.

\section{Conclusion}

The dynamics of the excited levels of the E\&e Jahn-Teller model, mapped onto the generalized Calogero-Moser gas of interacting pseudoparticles and developing in pseudotime, shows a complex interplay between the nonlinearity and fluctuations of the dynamical degrees of freedom. Numerical results of Section 2 on lattice dynamics and the probabilistic characteristics were interpreted, at least qualitatively, on the basis of the approximate analytical results of Section 3 .

In the intermediate range, $j \sim n_{r}$, the gCM dynamics implies a maximum degree of irregularity caused by the interference of three branches of the pseudocharges with growing range of interaction. We have found that the maximum mixing, and hence the broad maximum of stochasticity (irregularity) illustrated by the entropy of curvatures of the gCM trajectories (accelerations), is related to the new intermediate glassy phase of nucleation. This is thought to represent the growing kink-antikink chain structure. This scenario can be thought of for each level as an analogous to a series of local first order quantum phase transitions. In the intermediate droplet glassy phase, the phase of damped (anharmonic) oscillations dominating at $j<n_{r}$ and the lattice of interacting kink-antikink chains of the gCM trajectories dominating for $j>n_{r}$ coexist. As an alternative picture of this chain structure, we have recognised the kink-train lattice phase of the levels. These 

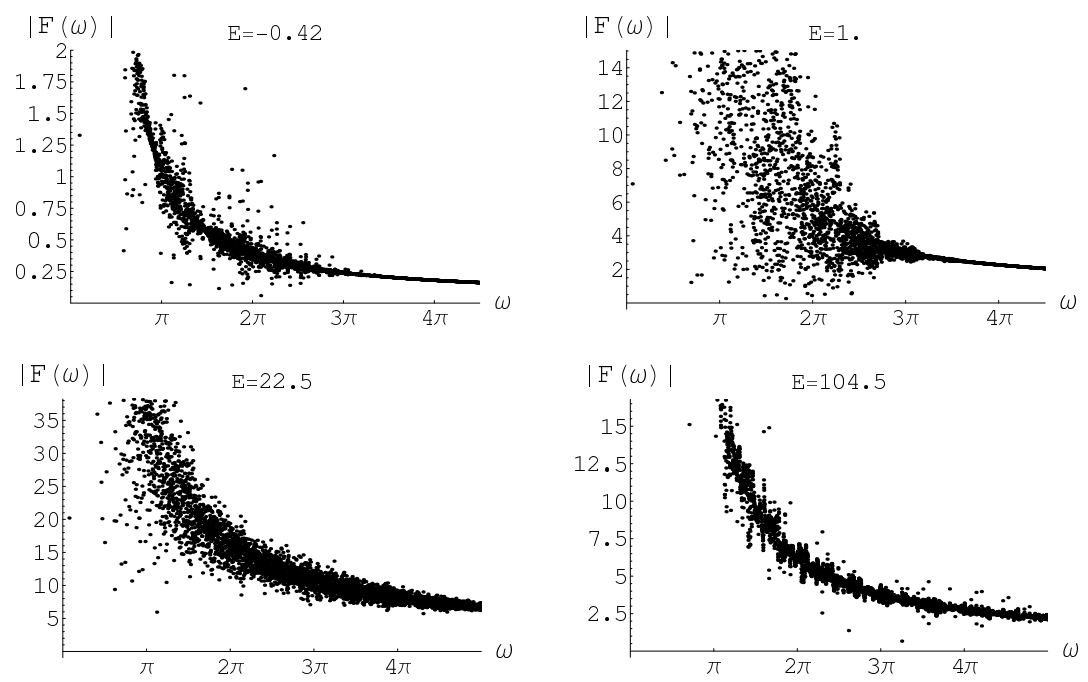

Fig. 8. Frequency spectrum of the $Q_{1}$ trajectory in semiclassical approximation for different values of total energy

move along straight lines broken by small intervals, where they undergo "collisions" (avoidings) with neighbour levels. They form a superlattice of excitations in the space of gCM pseudoparticles which we believe to represent a new quantum chaotic phase. The trajectories forming the kink train lattice of the kinks and antikinks move almost as free particles between collisions, with velocities of opposite sign distributed within two bands (Fig. 4a). The long-range order developed at large $\alpha$ stabilises the almost periodic kink-train lattice (Fig. 6). It is worth remembering that throughout this work we used the rotational quantum number $j$ as a parameter. Real spectral characteristics are cumulative ones including the contributions of all $j$ 's, where the multiple degeneracy in $j$ (level crossings) brings in components of regularity. Hence, real statistical properties present the mixing (coexistence) of contributions with prevailing higher degree of regularity or of those with more irregular nature. These are, however, highly nonuniversal.

The support of the project No. 202/06/0396 by the Grant Agency of the Czech Republic is greatly acknowledged as well as the support by the Grant Agency VEGA, Bratislava of the project No. 2/6073/26.

\section{References}

1. M. C. Gutzwiller, Chaos in Classical and Quantum Systems (Springer-Verlag, New York, 1990)

2. R. Ketzmerick, L. Hufnagel, F. Steinbach, M. Weiss, Phys. Rev. Lett. 83, 1214 (2000); M. Weiss, L. Hufnage, R. Ketzmerick, Phys. Rev. E 67, 046209 (2003); A. Bäcker, R. Ketzmerick, A. G. Monastra, Phys. Rev. E 75, 066204 (2007)

3. E. Majerníková, S. Shpyrko, J. Phys.: Condens. Matter 15, 2137 (2003)

4. P. Pechukas, Phys. Rev. Lett. 51, 943 (1983)

5. T. Yukawa, Phys. Rev. Lett. 54, 1883 (1985); T.Yukawa, Phys. Lett. A 116, 227 (1986)

6. K. Nakamura, M. Lakshmanan, Phys. Rev. Lett. 57, 1661 (1986); Phys.Rev.Lett. 57, 2772 (1986)
7. K. Nakamura, Quantum Chaos - A New Paradigm of Nonlinear Dynamics (Cambridge University Press, Cambridge, 1993)

8. X. Yang, J. Burgdörfer, Phys. Rev. Lett. 66, 982 (1991); Phys. Rev. A 462295 (1992)

9. H. Ishio, K. Nakamura, Phys. Rev. A 46, R2193 (1992)

10. H. Yamasaki, Y. Natsume, A. Terai, K. Nakamura, Phys. Rev. E 68, 046201 (2003); J. Phys. Soc. Jpn, 73, 1415 (2004)

11. E. Majerníková, S. Shpyrko, Phys. Rev. E 73, 066215 (2006)

12. P. Gaspard, S. A. Rice, H. J. Mikeska, K. Nakamura, Phys. Rev. A 42, 4015 (1990)

13. E. Majerníková, S. Shpyrko, Phys. Rev. E 73, 057202 (2006)

14. E. Majerníková, S. Shpyrko, J. Phys. A: Math. Theor. 41, $155102(2008)$

15. K. Nakamura, M. Lakshmanan, P. Gaspard, S.A. Rice, Phys.Rev. A 46, 6311 (1992)

16. H. C. Longuet-Higgins, U. Opik, M. H. L. Pryce, Proc. Roy. Soc. London, A 244, 1 (1958)

17. M. C. M. O'Brien, Proc. Roy. Soc.London, A 281, 323 (1964)

18. E.Majerníková, V.Majerník, S.Shpyrko, Eur. Phys. J. B 38, 25 (2004)

19. J. S. Langer, Ann. Phys. N.Y. 41108 (1967).

20. P. Lal, Phys. Lett. A 114, 410 (1986)

21. P. Gaspard, S. A. Rice, K. Nakamura, Phys. Rev. Lett. 63, 930 (1989)

22. J. Zakrzewski, A. Buchleitner, D. Delande, Z. Phys. B 103, 115 (1997)

23. R. Rajaraman, Solitons and Instantons (North-Holland Publ. Comp., Amsterdam-New York-Oxford 1982)

24. P. Anninos, S. Oliveira, R. A. Matzner, Phys. Rev. D 44, 1147 (1991)

25. D. K. Campbell, M. Peyrard, Physica D 19, 165 (1986)

26. R. H. Goodman, R. Haberman, Phys. Rev. Lett. 98, 104103 (2007)

27. Z. Fei, Y. S. Kivshar, L. Vázquez, Phys. Rev. A 45, 6019 (1992); Phys.Rev. A 46, 5214 (1992)

28. S. V. Dmitriev, P. G. Kevredikis, Y. S. Kivshar, e-print arxiv:nlin/0806.1152 